\title{
Computational View of $\beta$-Asarone-Human Serum Albumin Interaction
}

\author{
Md. Zahirul Kabir ${ }^{1, *}$, Saharuddin B. Mohamad ${ }^{2,3}$, Noridayu Omer ${ }^{4}$, Yeun-Mun Choo ${ }^{4}$, \\ Saad Tayyab $1,3, *$ iD
}

1 Biomolecular Research Group, Biochemistry Programme, Institute of Biological Sciences, Faculty of Science, Universiti Malaya, Kuala Lumpur, Malaysia; mmbzahirulkabir@gmail.com (M.Z.K.); saadtayyab2004@um.edu.my (S.T.);

2 Bioinformatics Programme, Institute of Biological Sciences, Faculty of Science, Universiti Malaya, Kuala Lumpur, Malaysia; saharuddin@um.edu.my (S.B.M.);

3 Centre of Research for Computational Sciences and Informatics for Biology, Bioindustry, Environment, Agriculture and Healthcare, Universiti Malaya, Kuala Lumpur, Malaysia; saadtayyab2004@um.edu.my (S.T.); saharuddin@um.edu.my (S.B.M.);

4 Department of Chemistry, Faculty of Science, Universiti Malaya, 50603, Kuala Lumpur, Malaysia; norii@um.edu.my (N.O.); ymchoo@um.edu.my (Y.M.C.);

* Correspondence: mmbzahirulkabir@gmail.com (M.Z.K.); saadtayyab2004@um.edu.my (S.T.);

Scopus Author ID 57114390900 (M.Z.K.)

7004517303 (S.T.)

Received: 17.07.2021; Revised: 25.08.2021; Accepted: 29.08.2021; Published: 18.10.2021

Abstract: $\beta$-Asarone (BAS), a bioactive phytochemical from the medicinal herb, Acorus calamus Linn., has shown many pharmacological activities. Computational docking studies unveiled the interaction site of BAS on the human plasma carrier, albumin. The primary binding arrangement of BAS was placed at Sudlow's Site I of HSA, which is pinpointed in subdomain IIA of albumin. Hydrophobic and van der Waals forces together with hydrogen bonds appear to secure the BAS-albumin complex. The BAS at Site I was surrounded by more hydrophobic and polar residues than those seen at Site II, as evidenced by LigPlot+. Therefore, the interaction between BAS and albumin at Site I seems to be comparatively more stable owing to more vital interactions.

Keywords: $\beta$-asarone; human serum albumin; drug-albumin interaction; computational docking; cluster analysis.

(C) 2021 by the authors. This article is an open-access article distributed under the terms and conditions of the Creative Commons Attribution (CC BY) license (https://creativecommons.org/licenses/by/4.0/).

\section{Introduction}

The $\beta$-asarone (1-propenyl-2,4,5-methoxybenzol) (BAS) with known chemical structure (Figure 1) is obtained from the medicinal herb, Acorus calamus Linn.[1]. Different pharmacological activities associated with BAS are anti-inflammatory, antiepileptic, antifungal, and antidepressant activities [2-5]. Several studies have reported that BAS functions as a promising anticancer agent in treating various carcinomas, viz., lung, gastric, hepatocellular, and colorectal cancers [6-9]. Due to its ability to cross the blood-brain barrier, BAS is frequently distributed in the cerebral tissue [10]. BAS has also shown positive results for treating neurodegenerative diseases, viz., Alzheimer's and Parkinson's diseases, depressive disorder, and ischemic stroke [5, 11-12]. 
<smiles>C/C=C/c1cc(OC)c(OC)cc1OC</smiles>

Figure 1. The chemical structure of BAS.

Drug-plasma protein binding influences a drug's transportation and distribution to its specific target locations [13,14]. Such interaction significantly impacts the drug's pharmacokinetics, such as enhanced in vivo half-life, prolonged efficacy, improved solubility, fast elimination, and reduced toxicity [15-17]. Therefore, the molecular interaction study between a drug and the protein is vital. Both experimental and in silico techniques are being used to characterize ligand-protein interactions. In silico analysis allows the first-hand interpretation of the preferred binding fashion of the drug to the protein's binding site at the atomic level [18-25]. However, these results need to be validated by experimental approaches. Albumin, present in human serum (HSA), is primarily responsible for carrying many drug molecules to their target organs throughout the bloodstream [15]. The drug's binding to albumin is expedited owing to the existence of two drug-binding sites, i.e., Sudlow's Sites I and II, placed in subdomains IIA and IIIA, respectively [26,27]. Several recent reports have shown the binding preference of various ligands to HSA at either of these two ligand-binding sites [28-33]. This paper describes the anticipated binding fashion and interaction of BAS with HSA at the atomic level using molecular docking assessments.

\section{Methods}

\subsection{Molecular docking.}

The computational docking simulation required the three-dimensional structures of BAS and HSA. BAS structure was generated using Avogadro software and optimized with the MMF94 force field [34,35]. Meanwhile, the PDB website was used to obtain the HSA structure (PDB ID: 1BM0). Optimization of HSA configuration involved the discharge of crystallized water, placement of polar hydrogens, as well as Kollman charges via AutoDockTools [36]. The docking simulations of BAS at the HSA Sites I and II were conducted independently with 100 search runs utilizing the Lamarckian genetic algorithm. The grid box was centralized at Site I (x: 35.36, y: 32.41, z: 36.46) and Site II (x: 14.42, y: 23.55, z: 23.31) separately with a dimension of $70 \times 70 \times 70$ and $0.375 \AA$ spacing. Other docking parameters were fixed as mutation (0.02), crossover operator weights (0.8), and elitism (1). The analysis of molecular docking results involved clustering the runs based on the $2.0 \AA$ root-mean-squared deviation (RMSD). Further interaction and visualisation were done utilizing UCSF Chimera and LigPlot+ [37,38].

\section{Results and Discussion}

\subsection{Molecular docking results.}

The atomic interactions generated between BAS and HSA can be predicted and visualized through molecular docking simulation. The independent site-specific docking 
simulation also enabled the identification of BAS binding priority on HSA Sudlow's binding sites. Based on the docking cluster analyses (Figure 2), Sites I and II were associated with overall 5 and 8 multi-member conformational clusters, respectively. The maximum populated cluster on Site I comprised 68 members $\left(\overline{\mathrm{X}}=-25.7 \mathrm{~kJ} \mathrm{~mol}^{-1}\right)$, whereas Site II included 49 members $\left(\overline{\mathrm{X}}=-17.2 \mathrm{~kJ} \mathrm{~mol}^{-1}\right)$. Meanwhile, the least calculated binding energy of BAS at Site I was $-26.9 \mathrm{~kJ} \mathrm{~mol}^{-1}$ compared to $-18.2 \mathrm{~kJ} \mathrm{~mol}^{-1}$ at Site II. All these initial analyses stated that the preference of BAS was leaning towards Site I of the protein.

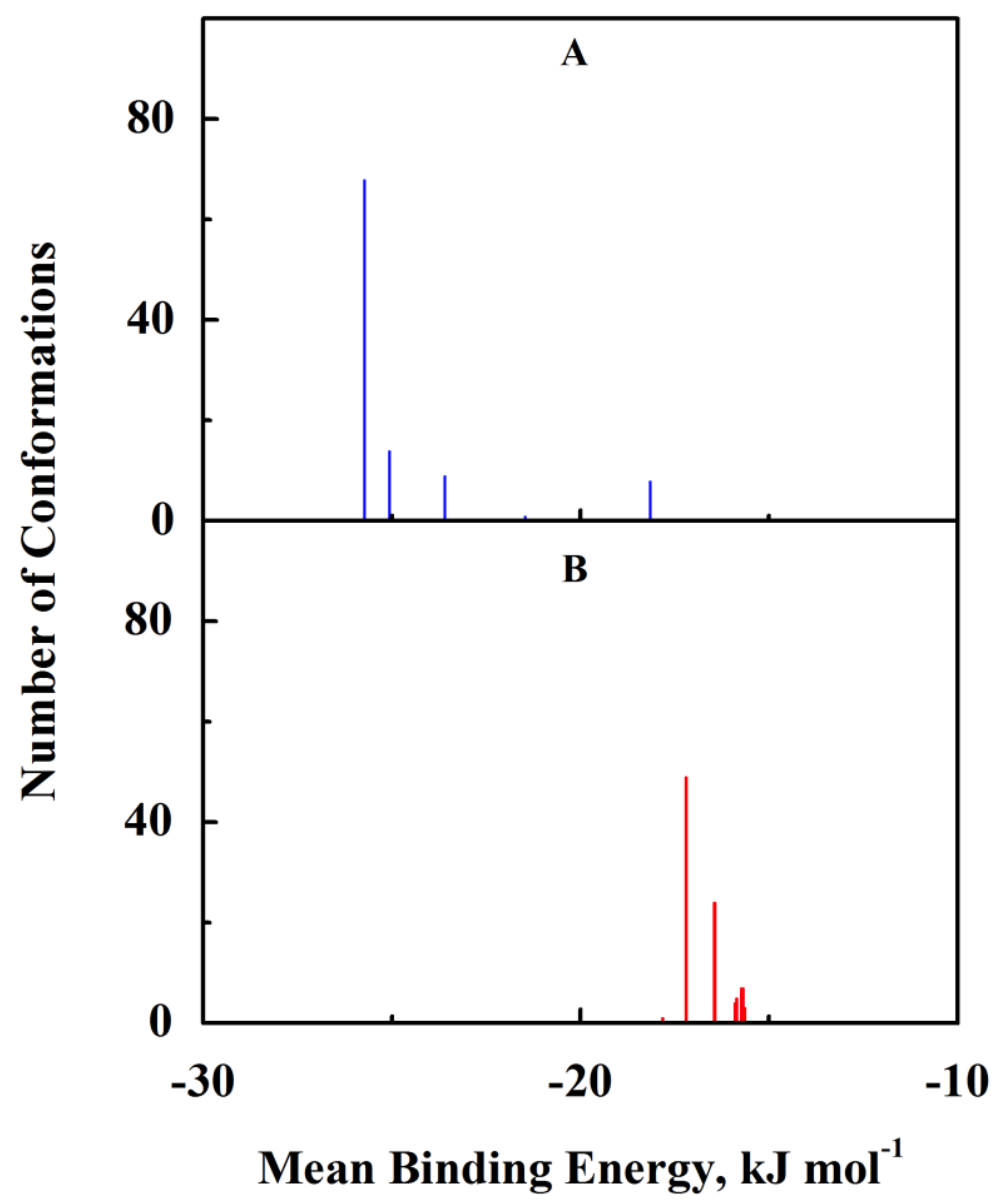

Figure 2. The cluster analyses of 100 AutoDock docking runs (RMSD $=2.0 \AA$ ) of BAS to the drug-binding sites, i.e., (A) Site I and (B) Site II of HSA (PDB: 1BM0).

Further analyses were accomplished by utilizing the lowest binding energy complex structures of BAS and albumin at Sites I and II (Figure $3 \mathrm{~A}-\mathrm{C}$ ). The investigation revealed that two H-bonds were formed when BAS was bound at Site I, while no hydrogen bond involvement was seen at Site II (Table 1). The surrounding pocket contributing to the polar and hydrophobic interactions (Figure 4A and 4B) showed engagement of many residues in this interaction when BAS was bound at Site I compared to Site II. The presence of hydrogen bonds plus other noncovalent interactions formed between BAS and Site I of HSA would have increased the complex stability. Overall, these analyses revealed the capability of BAS forming a stable complex while it is bound at Site I of HSA compared to Site II, hence favored its binding choice towards Site I of the protein. 


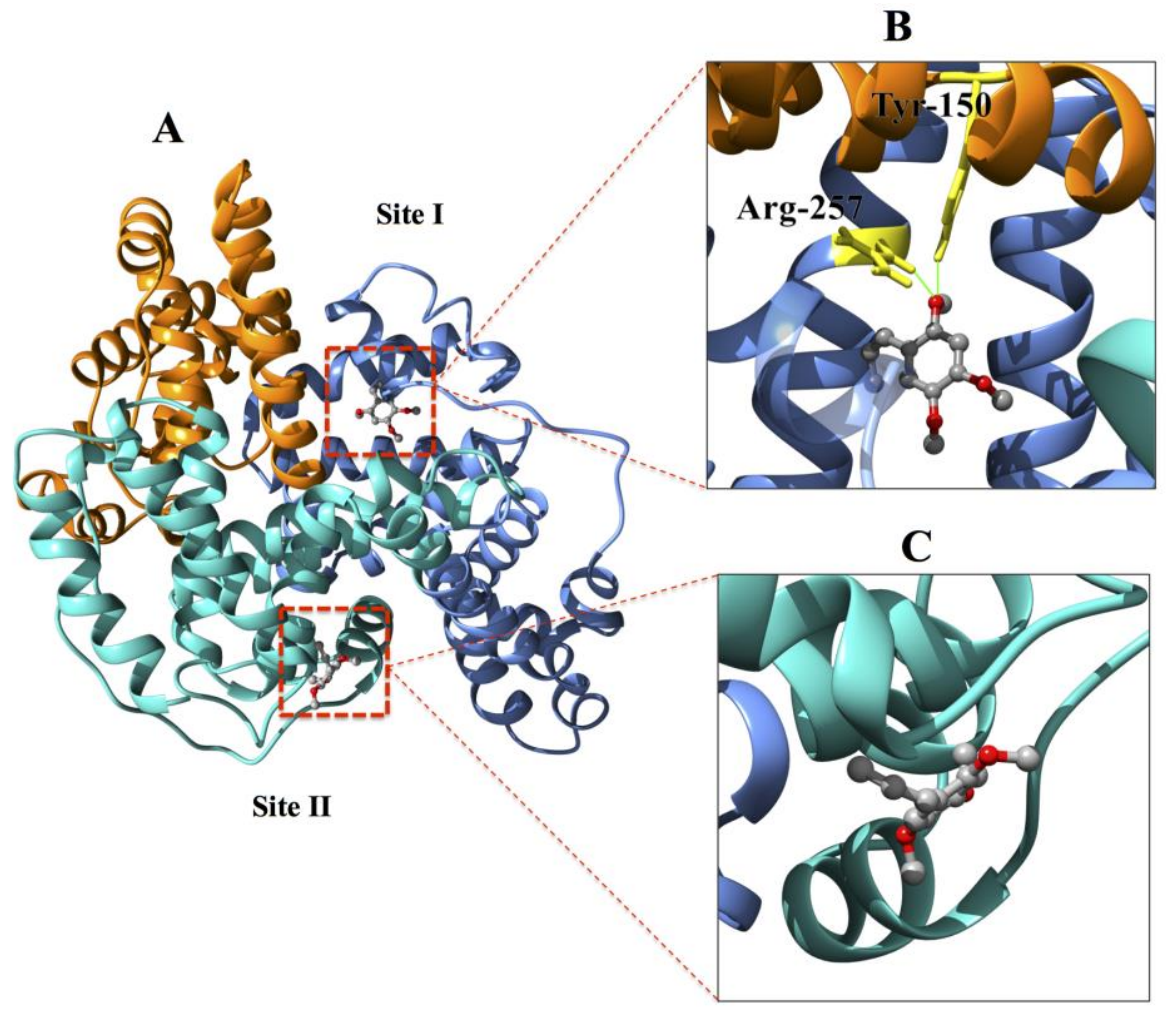

Figure 3. (A) Binding alignment of BAS (rendered in the sticks) in albumin binding sites I and II on the basis of least binding energy. Different domains I, II, and III of HSA are represented in orange, sky blue, and green colors, respectively. The enlarged view of the binding sites showed hydrogen bonds (green lines) within the protein's amino acid residues (rendered in the yellow stick) and BAS at Site I (B) and Site II (C) of HSA.

Table 1. Anticipated hydrogen bonds between atoms of amino acid residues of HSA and BAS at Sites I and II.

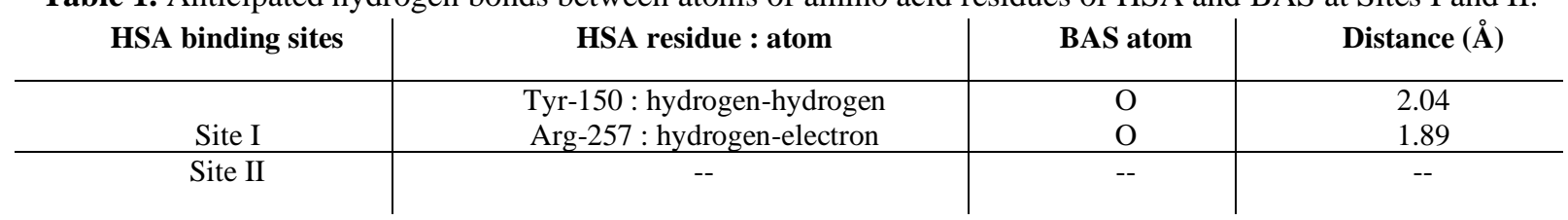

A

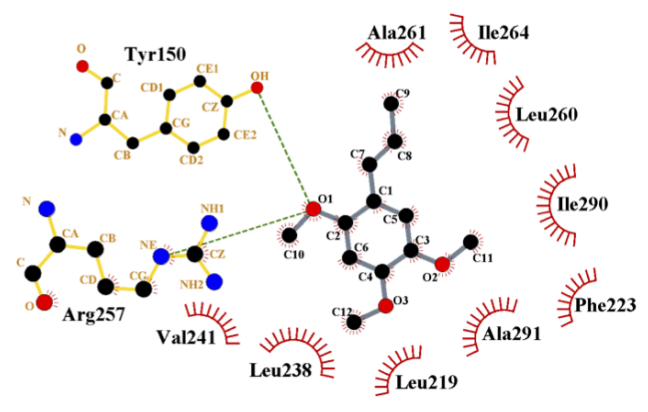

B

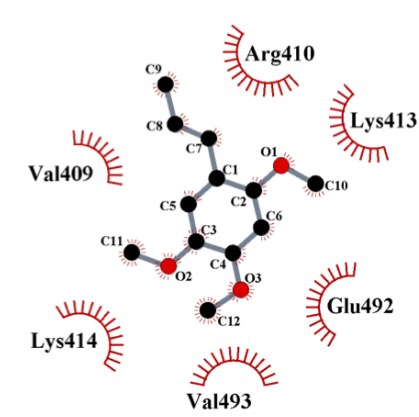

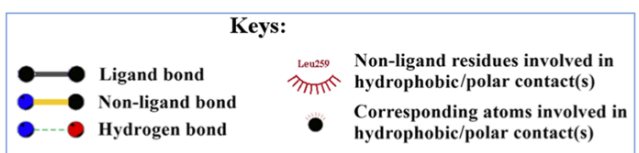

Figure 4. LigPlot+ maps present the hydrophobic and polar interactions between atoms of BAS and the amino acid residues of HSA at Site I (A) and Site II (B).

\section{Conclusions}

The findings obtained from our study indicated that the locus of BAS binding site on HSA was predicted at Site I (subdomain IIA). The stabilizing forces for the formed complex 
were found to be hydrophobic and polar interactions along with $\mathrm{H}$ bonds. The orientation of BAS bounded at Sudlow's Site I of HSA permitted substantial binding strength energy owing to increased non-covalent interactions and subsequently a stable complexation.

\section{Funding}

The Universiti Malaya Frontier Research Grant (FRG) 2017 (FG025-17AFR) provided financial support to carry out this work.

\section{Acknowledgments}

We thank the Dean, Faculty of Science, and the Head, Institute of Biological Sciences, Universiti Malaya, for providing the necessary facilities.

\section{Conflicts of Interest}

The authors have not identified competing for fiscal concerns or personal connections that could have emerged to affect the study reported here.

\section{References}

1. Chellian, R.; Pandy, V.; Mohamed, Z. Pharmacology and toxicology of $\alpha$ - and $\beta$-asarone: A review of preclinical evidence. Phytomedicine 2017, 32, 41-58, https://doi.org/10.1016/j.phymed.2017.04.003.

2. Lim, H.W.; Kumar, H.; Kim, B.W.; More, S.V.; Kim, I.W.; Park, J.I.; Park, S.Y.; Kim, S.K.; Choi, D.K. $\beta$ asarone (cis-2,4,5-trimethoxy-1-allyl phenyl), attenuates pro-inflammatory mediators by inhibiting NF-kB signaling and the JNK pathway in LPS activated BV-2 microglia cells. Food Chem. Toxicol. 2014, 72, 265272, https://doi.org/10.1016/j.fct.2014.07.018.

3. Fang, Y.Q.; Fang, R.M.; Fang, G.L.; Jiang, Y.; Fu, S.Y. Effects of beta-asarone on expression of c-fos in kindling epilepsy rat brain. Zhongguo Zhong Yao Za Zhi. 2008, 33, 534-536.

4. Lee, J.Y.; Lee, J.Y.; Yun, B.S.; Hwang, B.K. Antifungal activity of $\beta$-asarone from rhizomes of Acorus gramineus. J. Agric. Food Chem. 2004, 52, 776-780, https://doi.org/10.1021/jf035204o.

5. Dong, H.; Gao, Z.; Rong, H.; Jin, M.; Zhang, X. $\beta$-asarone reverses chronic unpredictable mild stress-induced depression-like behavior and promotes hippocampal neurogenesis in rats. Molecules 2014, 19, 5634-5649, https://doi.org/10.3390/molecules19055634.

6. Wang, T.L.; Ouyang, C.S.; Lin, L.Z. $\beta$-asarone suppresses Wnt/ $\beta$-catenin signaling to reduce viability, inhibit migration/invasion/adhesion and induce mitochondria-related apoptosis in lung cancer cells. Biomed. Pharmacother. 2018, 106, 821-830, https://doi.org/10.1016/j.biopha.2018.07.009.

7. Wu, J.; Zhang, X.X.; Sun, Q.M.; Chen, M.; Liu, S.L.; Zhang, X.; Zhou, J.Y.; Zou, X. $\beta$-asarone inhibits gastric cancer cell proliferation. Oncol. Rep. 2015, 34, 3043-3050, https://doi:10.3892/or.2015.4316.

8. Roy, S.R.; Gadad, P.C. Effect of $\beta$-asarone on diethylnitrosamine-induced hepatocellular carcinoma in rats. Indian J. Health Sci. Biomed. Res. KLEU 2016, 9, 82-88, https://doi:10.4103/2349-5006.183687.

9. Liu, L.; Wang, J.; Shi, L.; Zhang, W.; Du, X.; Wang, Z.; Zhang, Y. $\beta$-asarone induces senescence in colorectal cancer cells by inducing lamin B1 expression. Phytomedicine 2013, 20, 512-520, https://doi.org/10.1016/j.phymed.2012.12.008.

10. Fang, Y.Q.; Shi, C.; Liu, L.; Fang, R.M. Pharmacokinetics of beta-asarone in rabbit blood, hippocampus, cortex, brain stem, thalamus and cerebellum. Pharmazie 2012, 67, 120-123.

11. Chen, Y.; Wei, G.; Nie, H.; Lin, Y.; Tian, H.; Liu, Y.; Yu, X.; Cheng, S.; Yan, R.; Wang, Q.; Liu, D.H.; Deng, W.; Lai, Y.; Zhou, J.H.; Zhang, S.X.; Lin, W.W.; Chen, D.F. $\beta$-asarone prevents autophagy and synaptic loss by reducing ROCK expression in asenescence-accelerated prone 8 mice. Brain Res. 2014, 1552, 41-54, https://doi:10.1016/j.brainres.2014.01.005.

12. Huang, L.; Deng, M.; He, Y.; Lu, S.; Fang, Y. $\beta$-asarone increases MEF2D and TH levels and reduces $\alpha$ synuclein level in 6-OHDA-induced rats via regulating the HSP70/MAPK/MEF2D/Beclin-1 pathway: Chaperone-mediated autophagy activation, macroautophagy inhibition and HSP70 up-expression. Behav. Brain Res. 2016, 313, 370-379, https://doi:10.1016/j.bbr.2016.07.028. 
13. Barreca, D.; Lagana, G.; Bruno, G.; Magazu, S.; Bellocco, E. Diosmin binding to human serum albumin and its preventive action against degradation due to oxidative injuries. Biochimie 2013, 95, 2042-2049, https://doi:10.1016/j.biochi.2013.07.014.

14. Tesseromatis, C.; Alevizou, A. The role of the protein-binding on the mode of drug action as well the interactions with other drugs. Eur. J. Drug Metab. Pharmacokinet. 2008, 33, 225-230, https://doi.org/10.1007/BF03190876.

15. Peters, T. All about albumin: Biochemistry, genetics, and medical applications; San Diego, CA: Academic Press 1996, https://doi.org/10.1016/B978-0-12-552110-9.X5000-4.

16. Kragh-Hansen, U. Molecular and practical aspects of the enzymatic properties of human serum albumin and of albumin-ligand complexes. Biochim. Biophys. Acta 2013, 1830, 5535-5544, https://doi.org/10.1016/j.bbagen.2013.03.015.

17. Tayyab, S.; Feroz, S.R. Serum albumin: Clinical significance of drug binding and development as drug delivery vehicle. Adv Protein Chem Struct Biol. 2021, 123, 193-218, https://doi.org/10.1016/bs.apcsb.2020.08.003.

18. Siddiqui, S.; Ameena, F; Rehman, S.; Sarwar, T; Tabish, M. Studying the interaction of drug/ligand with serum albumin. J. Mol. Liq. 2021, 336, 116200, https://doi.org/10.1016/j.molliq.2021.116200.

19. Kılıç, S.; Andaç, M.; Denizli, A. Binding modes of cibacron blue with albumin in affinity chromatography using docking tools. Int. J. Biol. Macromol. 2021, 183, 110-118, https://doi.org/10.1016/j.ijbiomac.2021.04.142.

20. Kooravand, M.; Asadpour, S.; Haddadi, H.; Farhadian, S. An insight into the interaction between malachite green oxalate with human serum albumin: Molecular dynamic simulation and spectroscopic approaches. $J$. Hazard. Mater. 2021, 407, 124878, https://doi.org/10.1016/j.jhazmat.2020.124878.

21. Zhang, S.; Gan, R.; Zhao, L.; Sun, Q.; Xiang, H.; Xiang, X.; Zhao, G.; Li, H. Unveiling the interaction mechanism of alogliptin benzoate with human serum albumin: Insights from spectroscopy, microcalorimetry, and molecular docking and molecular dynamics analyses. Spectrochim. Acta A Mol. Biomol. Spectrosc. 2021, 246, 119040, https://doi.org/10.1016/j.saa.2020.119040.

22. Zhang, H.; Zhang, Y.; Huang, Y.; Wu, L.; Guo, Q.; Wang, Q.; Liang, L.; Nishinari, K.; Zhao, M. Interaction between bovine serum albumin and chitooligosaccharides: I. Molecular mechanism. Food Chem., 2021, 358, 129853, https://doi.org/10.1016/j.foodchem.2021.129853.

23. Sharma, V.; Sharma, A.K. An in-silico approach for designing a potential antagonistic molecule targeting $\beta_{2-}$ adrenoreceptor having therapeutic significance. Lett. Appl. NanoBioScience 2021, 10, 2063-2069, https://doi.org/10.33263/LIANBS101.20632069.

24. Lauro, F.V; Francisco, D.C.; Maria, L.R.; Marcela, R.N.; Virginia, M.A.M.; Alejandra, G.E.; Regina, C.C.; Yazmin, O.A. Design and synthesis of five cyclobuta-1,3-dien-1-yl- steroid derivatives to evaluate their theoretical activity against covid-19. Lett. Appl. NanoBioScience 2020, 9, 1177-1187, https://doi.org/10.33263/LIANBS93.11771187.

25. Patel, V.; Shaha, J.; Gupta A.K. Design and in-silico study of bioimaging fluorescence graphene quantum dot-bovine serum albumin complex synthesized by diimide-activated amidation. Comput. Biol. Chem. 2021, 93, 107543, https://doi.org/10.1016/j.compbiolchem.2021.107543.

26. Kragh-Hansen, U.; Chuang, V.T.G.; Otagiri, M. Practical aspects of the ligand-binding and enzymatic properties of human serum albumin. Biol. Pharm. Bull. 2002, 25, 695-704, https://doi.org/10.1248/bpb.25.695.

27. Sudlow, G.; Birkett, D.J.; Wade, D.N. The characterization of two specific drug binding sites on human serum albumin. Mol. Pharmacol. 1975, 11, 824-832.

28. Tayyab, S.; Magesvaran, M.K.A.; Kabir, M.Z.; Ridzwan, N.F.W; Mohamad, S.B. Biophysical and computational view on the in vitro combination between an anticancer drug, saracatinib and human serum albumin. J. Biomol. Struct. Dyn. 2021, 39, 3565-3575, https://doi.org/10.1080/07391102.2020.1766571.

29. Kabir, M.Z.; Benbekhti, Z.; Ridzwan, N.F.W.; Merrouche, R.; Bouras, N.; Mohamad, S.B.; Tayyab, S. Biophysical and in silico investigations of the molecular association between a potent RNA polymerase inhibitor, thiolutin and human serum albumin. $J$ Mol Liq. 2020, 303, 112648, https://doi.org/10.1016/j.molliq.2020.112648.

30. Roslan, A.A.; Mohamad, S.B.; Tayyab, S. Docking evaluation of the interaction between green tea active ingredient, L-theanine and human serum albumin. Natl. Acad. Sci. Lett. 2021, 44, 17-19, https://doi.org/10.1007/s40009-020-00949-5. 
31. Kandandapani, S.; Kabir, M.Z.; Ridzwan, N.F.W; Mohamad, S.B.; Tayyab, S. Biomolecular interaction mechanism of an anticancer drug, pazopanib with human serum albumin: A multi-spectroscopic and computational approach. J. Biomol. Struct. Dyn. 2021, https://doi.org/10.1080/07391102.2021.1911850.

32. Liang, Y.; Xu, L.; Yang, H.; Xu, W.; Hu, R.; Fan, X.; Liu, Y. Analysis on the interaction and binding properties of daphnoretin and human serum albumin in the presence of cisplatin: multi-spectroscopic methods and docking simulation. Eur. J. Pharm. Sci. 2021, 159, 105723, https://doi.org/10.1016/j.ejps.2021.105723.

33. Salim, M.M.; Sharkasy, M.E.E.; Walash, B.M. Multi-spectroscopic and molecular docking studies for binding interaction between fluvoxamine and human serum albumin. Spectrochim. Acta A Mol. Biomol. Spectrosc. 2021, 252, 119495, https://doi.org/10.1016/j.saa.2021.119495.

34. Bhattacharyya, J.; Bhattacharyya, M.; Chakrabarty, A.S.; Chaudhuri, U.; Poddar, R.K. Interaction of chlorpromazine with myoglobin and hemoglobin: A comparative study. Biochem. Pharmacol. 1994, 47, 2049-2053, https://doi:10.1016/0006-2952(94)90080-9.

35. Hanwell, M.D.; Curtis, D.E.; Lonie, D.C.; Vandermeersch, T.; Zurek, E.; Hutchison, G.R. Avogadro: An advanced semantic chemical editor, visualization, and analysis platform. J. Cheminformatics 2012, 4, 1-17, https://doi.org/10.1186/1758-2946-4-17.

36. Halgren, T.A. Merck molecular force field. I. Basis, form, scope, parameterization, and performance of MMFF94. J. Comput. Chem. 1996, 17, 490-519, https://doi.org/10.1002/(SICI)1096987X(199604)17:5/6<490::AID-JCC1>3.0.CO;2-P.

37. Morris, G.M.; Huey, R.; Lindstrom, W.; Sanner, M.F.; Belew, R.K.; Goodsell, D.S.; Olson, A.J. AutoDock4 and AutoDockTools4: Automated docking with selective receptor flexibility. J. Comput. Chem. 2009, 30, 2785-2791, https://doi.org/10.1002/jcc.21256.

38. Pettersen, E.F.; Goddard, T.D.; Huang, C.C.; Couch, G.S.; Greenblatt, D.M.; Meng, E.C.; Ferrin, T.E. UCSF Chimera - A visualization system for exploratory research and analysis. J. Comput. Chem. 2004, 25, 16051612, https//doi.org/10.1002/jcc.20084. 